\title{
Colostomia continente com esfíncter artificial de silicone. Estudo em cães ${ }^{1}$
}

\author{
Edmundo Guilherme de Almeida Gomes ${ }^{2}$ \\ Carlos Teixeira Brandt ${ }^{3}$ \\ Mario Jorge Jucá ${ }^{4}$ \\ Hunaldo Lima de Menezes ${ }^{5}$
}

Gomes EGA, Brandt CT, Jucá MJ, Menezes HL. Colostomia continente com esfíncter artificial de silicone: estudo em cães. Acta Cir Bras [serial online] 2003 Nov-Dez;18(6). Disponível em URL: http://www.scielo.br/acb.

RESUMO - Objetivo: A presente investigação teve como objetivo implantar um esfíncter artificial de silicone ( C C - Silimed ${ }^{\circledR}$ ) em colostomias terminais, em cães, e analisar a continência às fezes sólidas e líquidas, como também as complicações advindas do procedimento. Métodos: Foram utilizados 13 cães mestiços, 11 machos e 2 fêmeas, com peso que variou entre 16 e $30 \mathrm{~kg}$, média de $22,5 \pm 4,3 \mathrm{~kg}$. Foram construídas colostomias terminais dotadas do C C Silimed $^{\circledR}$, que eram ativados por um período de 8 horas diariamente. Os animais eram observados durante 15 semanas. A continência alcançada era demonstrada radiologicamente através de enema baritado. Resultados: A pressão no sistema variou entre 60 e $80 \mathrm{~cm}$ de água, média de $74,8 \pm 5,0 \mathrm{~cm}$. O volume de fluido que era introduzido no reservatório oscilou entre 5 e $8 \mathrm{ml}$, média de $6,9 \pm 0,8 \mathrm{ml}$. A continência foi alcançada em todos os animais, mas ocorreu escape fecal esporádico nos cães, durante esforços físicos. Infecção da ferida operatória aconteceu em três cães ( $23,1 \%$ ) e obstrução intestinal em ( 7,7\% ). Conclusão: O esfíncter artificial de silicone ( C C-Silimed $\left.{ }^{\circledR}\right)$ mostrou-se efetivo na continência para fezes sólidas e líquidas. Assim sendo, abre uma expectativa para seu emprego clínico, em tempo futuro, em indivíduos portadores de colostomia definitiva.

DESCRITORES - Colostomia. Incontinência fecal. Esfíncter artificial.

\section{Introdução}

A confecção de um estoma resulta num impacto estético e psicológico, em decorrência das mudanças na imagem corporal, que levam a sensações de mutilação, invalidez, desgosto pelo fato de evacuar pelo abdômen, pela presença de vazamentos de gases e fezes, associados ao medo de ficar incapaz de se relacionar sexualmente, a diminuição da libido e a depressão, que geram uma série de obstáculos na reintegração e ajustamento à vida familiar, social e laborativa ${ }^{1}$.

Na tentativa de reabilitar e melhorar a qualidade de vida dos ostomizados, vários métodos foram elaborados para minimizar ou mesmo sanar as dificuldades. As bolsas com material plástico anti-odor, hipoalergênico, não tóxico e com alta adesividade. Os acessórios de diversos tipos e finalidades, representados por filtro, cinto elástico ajustável, oclusores e sistema de irrigação. Todos com o objetivo de tornarem as colostomias continentes ou, pelo menos, para reduzir o número de eliminações fecais, a produção de ruídos e odores, reabilitando o indivíduo ao convívio social e recuperando a sua capacidade produtiva ${ }^{2}$.

Na década de 70, surgiram os oclusores intestinais do tipo balão circunferencial e luminal. O primeiro, uma adaptação para ser utilizado no trato digestivo, a partir de um esfíncter urinário desenvolvido por Scott, Bradley e Timm ${ }^{3}$, constituído de materiais não biológicos, que obteve resultados funcionais satisfatórios em cães ileostomizados ${ }^{4}$. Pioneiramente, após novas modificações,
Christiansen e Lorentzen ${ }^{5}$ utilizaram a mecânica do esfíncter artificial de silicone em portadores de incontinência anal de origem neurogênica, como opção à derivação intestinal, e a continência alcançada para fezes sólidas e semi-sólidas foi considerada aceitável. Os luminais, que funcionam como tampão, tanto o magnético $^{6}$, como o sistema oclusor de colostomia ${ }^{7}$, mostraram-se ineficazes.

O reservatório ileal com mecanismo valvular desenvolvido em $1969^{8}$, precedeu outros ensaios, como o de reproduzir o reservatório retal e o aparelho esfincteriano, após ressecção abdominoperineal do reto, com uma colostomia perineal dotada de neoesfíncter confeccionado com a transposição do músculo grácil ${ }^{9}$, que depois foi estimulado eletricamente para desenvolver características de

\footnotetext{
1. Trabalho realizado DEPTO na Universidade Federal de Alagoas (UFAL). Prof. voluntário da Disc. de Coloproctologia - UFAL.

2. Mestre em Cirurgia da Universidade Federal de Pernambuco (UFPE), Prof. voluntário da Disc. de Coloproctologia - UFAL.

3. Prof. Titular de Clínica Pediátrica Cirúrgica da UFPE.

4. Prof. Adjunto, Doutor de Clínica Cirúrgica, CSAU, UFAL.

5. Mestre em Cirurgia da UFPE.
} 
músculo liso, mas os resultados foram insatisfatórios ${ }^{10}$. Posteriormente, surgiu a colostomia com transplante livre de seromuscular do cólon, que apresentou dificuldade para esvaziamento do conteúdo colônico. ${ }^{11}$

Portanto, a colostomia continente tem sido uma aspiração de pacientes e investigadores, contudo, ainda não foi desenvolvido um método simples, eficiente, acessível e com poucas complicações. $\mathrm{O}$ esfíncter artificial apresenta-se como uma alternativa atraente com os avanços obtidos nos últimos anos.

A presente investigação teve como objetivo analisar a continência às fezes sólidas e líquidas proporcionada pelo implante de um esfíncter artificial de silicone em colostomias terminais, como também as possíveis complicações advindas do procedimento.

\section{Métodos}

Foram utilizados 13 cães adultos, mestiços, 11 machos e 2 fêmeas, com peso corporal que variou entre 16 e $30 \mathrm{~kg}$, média de 22,5 $\pm 4,3 \mathrm{~kg}$ (Tabela 1), oriundos do Biotério Central da Universidade Federal de Alagoas, após o protocolo de pesquisa ter sido aprovado pela Comissão de Ética da UFAL.

O constrictor de cólon (CC Silimed ${ }^{\circledR}$ ) é constituído por um corpo reservatório composto por três camadas. A face interna, que fica em contato com a parede do cólon, é de espuma de poliuretano. A face externa com reforço de poliéster encapsulado em silicone, dotado de 4 lingüetas para fixação e um tubo de silicone, que une o corpo do dispositivo à outra extremidade onde está situada a válvula de controle. (Figura 1).

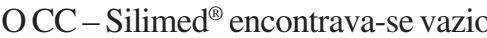
em situação de repouso, o que mantinha a colostomia aberta. Para promover a oclusão da alça intestinal, puncionava-se a válvula de controle, injetava-se água destilada que insuflava o reservatório, com volumes de água que variavam de 5 a $8 \mathrm{ml}$, produzindo uma pressão no sistema que era capaz de ocluir o estoma sem provocar isquemia.

Os animais no pré-operatório eram pesados e recebiam coleiras numeradas. A medicação pré-anestésica era a clorpromazina, na dose de $1 \mathrm{mg} / \mathrm{kg}$ endovenosa. A indução anestésica era realizada com a infusão venosa de tiopental sódico ( $20 \mathrm{mg} / \mathrm{kg}$ ). Os animais permaneciam com ventilação espontânea utilizando máscara de oxigênio. Em seguida, era promovida laparotomia mediana e confeccionada colostomia terminal esquerda, no cólon sigmóide, com sepultamento do coto distal. Durante o posicionamento do estoma, colocava-se o CC Silimed $^{\circledR}$ em torno da alça exteriorizada, que ficava localizado intra-cavitário, fixado no peritônio pelas lingüetas. Um túnel subcutâneo era produzido do orifício da colostomia até o flanco esquerdo, onde era posicionada a válvula de controle. (Figura 2).

Os cães, no pós-operatório, permaneciam com o dispositivo desativado por um período de 4 semanas. A avaliação da continência obtida com o constrictor de cólon iniciava-se na quinta semana com um período de insuflação de 2 horas diárias, que passava para 4 horas na sexta semana e 8 horas na sétima semana, permanecendo por mais 8 semanas. Isso ocorria até ter-se encontrado a pressão ideal para a oclusão da colostomia, utilizando uma coluna d'água para mensuração e não ultrapassava $80 \mathrm{~cm}$ de água. $\mathrm{O}$ enema opaco era utilizado no final da observação para caracterizar o resultado funcional da prótese, detectando a presença ou ausên- cia de vazamento da solução contrastada pelo estoma.

Os resultados das variáveis quantitativas foram expressos por suas médias e respectivos desvios padrão. O coeficiente de correlação de Pearson foi utilizado para avaliar possível correlação entre variáveis. Foi aceito o limite de $\mathrm{p}<0,05$ para rejeição da hipótese de nulidade.

\section{Resultados}

A pressão no sistema variou entre 60 e $80 \mathrm{~cm}$ de água, média de $74,8 \pm 5,0 \mathrm{~cm}$. Utilizou-se um volume de água para insuflar o reservatório que oscilou entre 5 e 8ml, média de 6,9 $\pm 0,8 \mathrm{ml}$ (Tabela $2 \mathrm{e}$ Figura 3).

O coeficiente de correlação de Pearson entre as variáveis, quantidade de fluido e pressão, foi r =0,8797. A expressão geométrica da correlação entre as duas variáveis e a equação de regressão linear que expressa a estimativa delas estão na Figura 4.

A continência com a implantação do CC - Silimed ${ }^{\circledR}$ foi alcançada em todos os animais. Entretanto, os cães apresentaram pequenos vazamentos esporádicos de fezes, tipo escape fecal, verificados durante esforços físicos na manipulação dos mesmos, mas esses eventos não ocorreram nos locais de confinamento. Para melhor avaliação da continência, principalmente às fezes líquidas, realizava-se um enema opaco, que mostrou ausência de vazamento da solução baritada pelo estoma (Figura 5).

Infecção de ferida operatória foi a complicação mais freqüente, tendo ocorrido em três ( $23,1 \%)$ animais. Um cão $(7,7 \%)$ apresentou obstrução intestinal na $11^{a}$ semana de avaliação, devido a aderências das alças do intestino delgado à prótese intra-peritoneal.

TABELA 1 - Peso dos cães

\begin{tabular}{llllllllllllll}
\hline $\mathrm{N}^{\circ}$ & $\mathrm{C} 1$ & $\mathrm{C} 2$ & $\mathrm{C} 3$ & $\mathrm{C} 4$ & $\mathrm{C} 5$ & $\mathrm{C} 6$ & $\mathrm{C} 7$ & $\mathrm{C} 8$ & $\mathrm{C} 9$ & $\mathrm{C} 10$ & $\mathrm{C} 11$ & $\mathrm{C} 12$ & $\mathrm{C} 13$ \\
\hline Peso \\
$(\mathrm{kg})^{*}$ & 23 & 25 & 27 & 24 & 22 & 16 & 17 & 30 & 20 & 23 & 18 & 19 & 28 \\
\hline * quilograma & & & & & & & & & & & \\
C=Cão \\
Média $=22,5 \mathrm{Kg}$
\end{tabular}




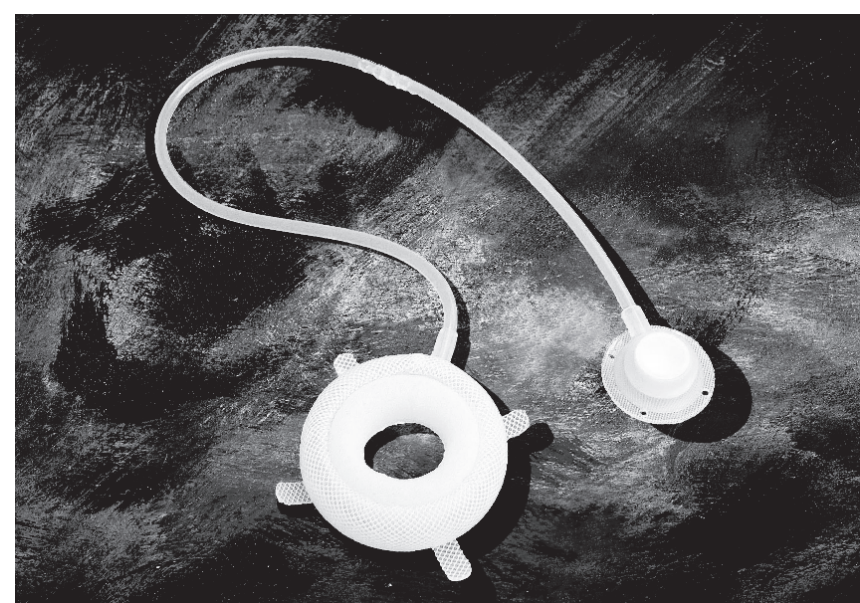

FIGURA 1 - Constrictor de cólon.

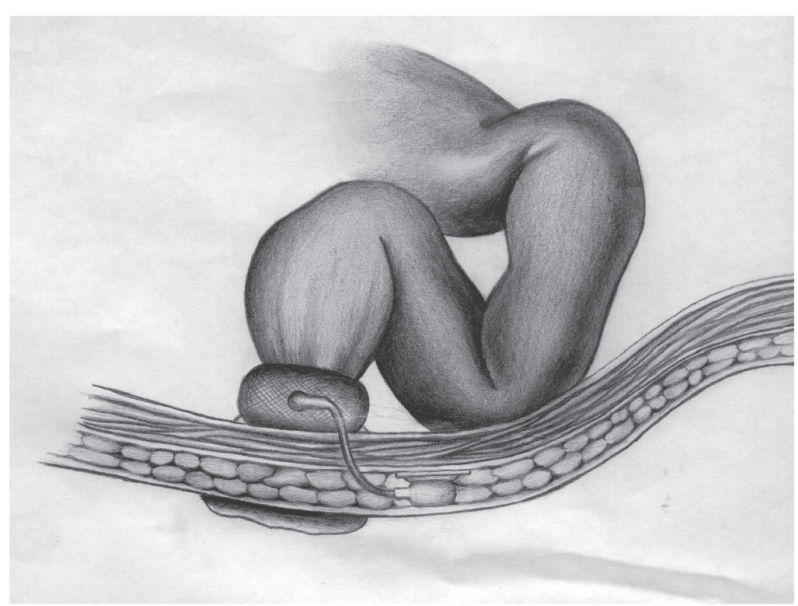

FIGURA 2 - Representação gráfica da localização intra-peritoneal

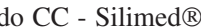

TABELA 2 - Relação das quantidades de fluido com as respectivas pressões no reservatório.

\begin{tabular}{ccc}
\hline $\mathbf{N}^{\mathbf{0}}$ do Cão & $\begin{array}{c}\text { Quantidade de } \\
\text { fluido(ml) }\end{array}$ & $\begin{array}{c}\text { Pressão do reservatório } \\
\left(\mathbf{c m} \text { de } \mathbf{H}_{2} \mathbf{O}\right)\end{array}$ \\
\hline 1 & 5,0 & 60 \\
2 & 7,0 & 76 \\
3 & 6,0 & 72 \\
4 & 7,5 & 75 \\
5 & 6,5 & 73 \\
6 & 7,0 & 74 \\
7 & 7,0 & 79 \\
8 & 7,5 & 77 \\
9 & 8,0 & 78 \\
10 & 6,5 & 75 \\
11 & 7,0 & 76 \\
12 & 7,0 & 77 \\
13 & 8,0 & 80 \\
& 6,9 & 74,8 \\
Média & 0,8 & 5,0 \\
Desvio-Padrão & 0,12 & 0,07 \\
\hline
\end{tabular}

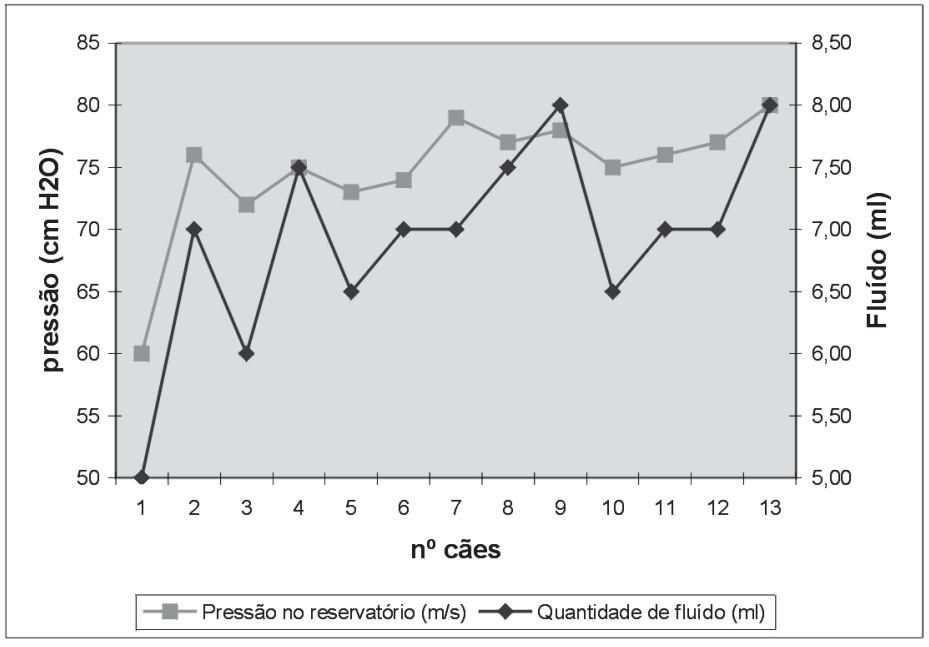

FIGURA 3 - Relação das quantidades de fluido com as respectivas pressões no reservatório.

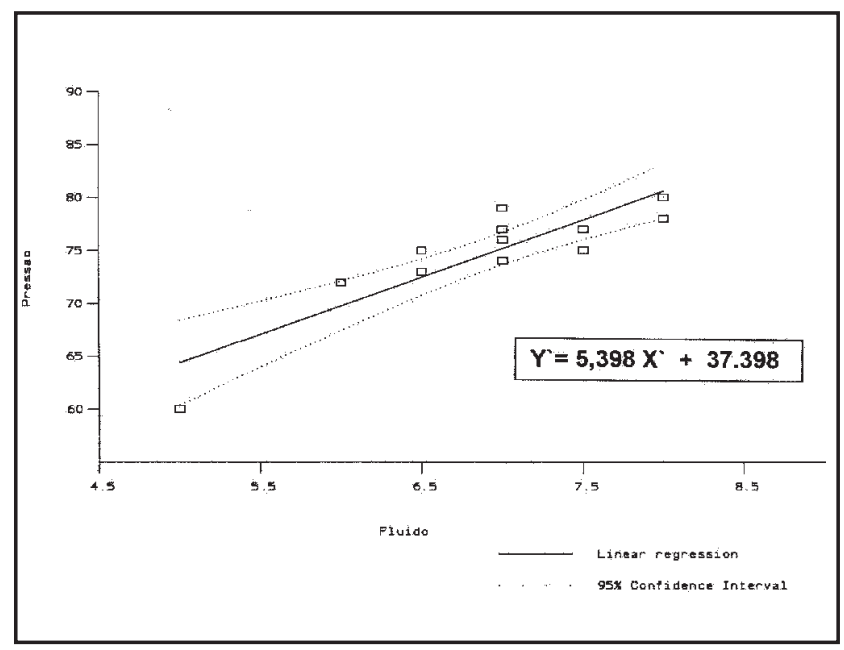

FIGURA 4 - Expressão da correlação entre a quantidade de fluido e a pressão. 


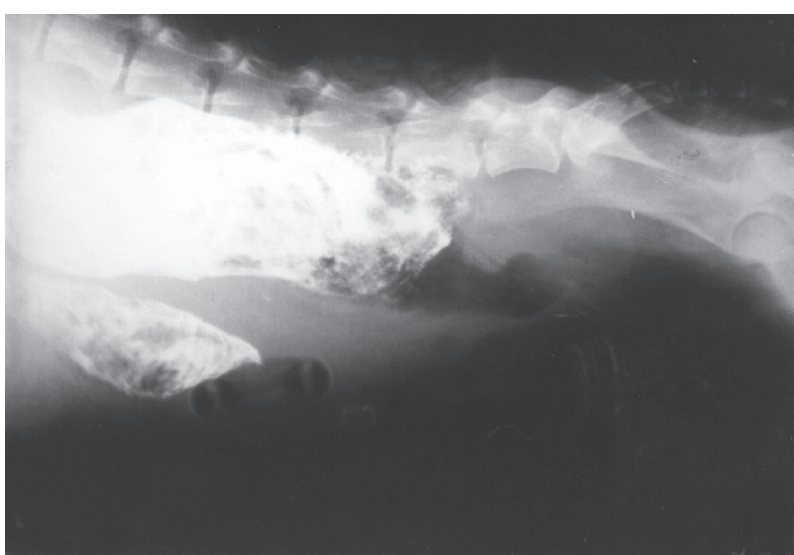

FIGURA 5 - Enema baritado demonstrando a continência às fezes líquidas produzida pelo CC - Silimed®.

\section{Discussão}

Uma colostomia definitiva, sem dúvida alguma, dá origem a um grande impacto na rotina diária do indivíduo, pois com a abolição de uma função importante do corpo, a capacidade de regular a eliminação dos excrementos fecais, associada a distorção da imagem corporal, acompanhada da mudança do funcionamento físico e dos cuidados pessoais, requerem uma habilidade de ajustamento para se chegar ao domínio da situação ${ }^{12}$.

A construção de estomas é um procedimento cirúrgico que está se tornando mais freqüente, contudo, os cuidados mudaram dramaticamente com a formação de profissionais qualificados, os estomoterapêutas, aliados a introdução de materiais de alta qualidade, que melhorou o manejo dos mesmos. No entanto, os estudos psicossociais revelaram que os problemas emocionais persistem, sendo representados pela diminuição dos contatos sociais, da libido e problemas relacionados ao trabalho. Todavia, existem três fatores que são considerados responsáveis pelas diferenças individuais, quais sejam, o médico, o sociodemográfico e o psicológico $^{12}$.

Esses fatores vêm sendo pesquisados a várias décadas na busca de uma solução viável para esses indivíduos. Com o surgimento dos materiais não biológicos empregados na fabricação de esfíncteres artificiais, além dos avanços divulgados nos últimos anos, obtidos com dispositivos especialmente desenhados para o tratamento da incontinência fecal, com a melhor adequação técnica, o equacionamento dos problemas hidráulicos, o fácil manuseio e a possibilidade de substituição ${ }^{13}$, têm demonstrado ser uma opção real para obtenção de continência em colostomias definitivas e é corroborada pelos bons resultados conseguidos nesta investigação em cães.

O esfíncter artificial de silicone ( CC - Silimed ${ }^{\circledR}$ ) demonstrou continência efetiva para fezes sólidas e líquidas, por um período de 8 horas diárias, durante 15 semanas. Fato demonstrado radiologicamente por enema baritado. Esse resultado é comparável a outros relatos que utilizaram cães ileostomizados, com um tempo maior de observação ${ }^{4,14}$. Os dois estudos clínicos em colostomias, um realizado por Heiblum e Cordoba ${ }^{15}$, o outro por Szinicz ${ }^{16}$, com períodos curtos de avaliação, obtiveram resultados funcionais parecidos. Todavia, os seguimentos a longo prazo jamais foram divulgados, o que impossibilita tecer comentários sobre os motivos do abandono do método.

Neste trabalho a pressão no sistema variou entre 60 e $80 \mathrm{~cm}$ de água e foi semelhante há outros autores que utilizaram de 70 a $100 \mathrm{~cm}$ de água ${ }^{4,14,16}$. Um modelo experimental diferente utilizou uma força eletromecânica que tracionava uma banda plástica para constringir o cólon, a continência para gás foi alcançada com valores acima de 59, $2 \mathrm{~cm}$ de água ${ }^{17}$. Todavia, a pressão sob o oclusor, ou pressão intraluminal, permanece 10 a $20 \mathrm{~cm}$ de água menor $^{14}$. Com cintilografia demonstrou-se que o fluxo sangüíneo na camada muscular e submucosa não sofreu alteração significativa, mas na mucosa houve redução do fluxo pela metade quando comparado com o controle, no entanto, foi suficiente para manter a viabilidade do segmento observadas alterações histopatológicas relacionadas com a isquemia ${ }^{4}$.

A complicação mais freqüente na presente investigação foi a infecção da ferida operatória ( $23,1 \%$ ), presente em estudos que utilizaram cães ileostomizados, um mostrou-se semelhante com $30 \%{ }^{4}$, e outro com uma taxa bem menor de $5,5 \%{ }^{14}$, compatível com a encontrada em pacientes submetidos a colostomia desprovida do esfíncter artificial, que foi de $7,2 \%{ }^{18}$. A erosão da pele não ocorreu neste relato, mas foi verificada quando o dispositivo ficou situado na parede abdominal do cão, que apresenta uma espessura inadequada para abrigá-lo. Essa complicação geralmente é acompanhada por infecção. Outra complicação observada neste ensaio foi a obstrução intestinal ( $7,7 \%$ ), que aconteceu por aderências firmadas entre o constrictor de cólon com o intestino delgado, fato descrito por outros trabalhos, com incidências de 20 e $27 \%^{4,14}$. O silicone é reativo dentro da cavidade peritoneal e pode gerar aderências envolvendo alças intestinais. Clinicamente ocorreu em 7,2\% dos indivíduos ${ }^{18}$

\section{Conclusão}

Os resultados obtidos neste ensaio experimental, utilizando um esfíncter artificial de silicone $\left(\mathrm{CC}-\right.$ Silimed $^{\circledR}$ ) em cães colostomizados, permitem concluir que o mesmo foi efetivo na continência para fezes sólidas e líquidas, tendo como complicação freqüente a infecção da ferida operatória e, quando implantado no interior da cavidade peritoneal, pode produzir aderências.

\section{Referências}

1. Oades-Souther D, Olbrisch ME Psychological adjustment to ostomy 
surgery. Rehab Psychol 1984; 29: 221-37.

2. Silva R, Silva TR, Crema E. Dispositivos e acessórios para estomas. In : Crema E, Silva R. Estomas : uma abordagem multidisciplinar. Uberaba: Pinti Editora; 1997. p 65-76.

3. Scott FB, Bradley WE, Timm GW. Treatment of urinary incontinence by implantable prosthetic sphincter. Urology 1973; 1: 252-9.

4. Delaney J, Broadie T, Timm G, Bradley W. A prosthetic sphincter for the gastrointestinal tract. J Surg Res 1974;16: 204-9.

5. Christiansen J, Lorentzen M. Implantation of artificial sphincter for anal incontinence: report of five cases. Dis Colon Rectum 1989;32: 432-6.

6. Feustel H, Hennig G. Kontinente kolostomie durch magnetverschluss. Dtsch Med Wschr 1975; 100: 1063-4.

7. Burcharth F, Ballan A, Kylberg F, Rasmussen SN. The colostomy plug: a new disposable device for a continent colostomy. Lancet 1986; 2: 1062-3.

8. Koch NG. Intra-abdominal "reservoir" in patients with permanent ileostomy: preliminary observation on a procedure resulting in fecal "continence" in five patients. Arch Surg 1969; 99: 223.

9. Simonsen OS, Stolf NAG, Aun F, Raia A, Habr-Gama A. Rectal sphincter reconstruction in perineal colostomies after abdominoperineal resection for cancer. $\mathrm{Br}$ J Surg 1976; 63: 389-91.

10. Cavina E, Seccia M, Evangelista G. Construction of a continent perianal colostomy by using electrostimulated gracilis muscle after abdominoperineal resection. Personal technique and experience with 32 cases. Ital J Surg Sci 1987; 4: 305-14

11. Schmidt E. The continent colostomy. World J Surg 1982; 6: 805-9.

12. Bekkers MJTM, van Knippenberg FCE, van den Borne HW, van Berge Henegouwen GP. Prospective evaluation of psychosocial adaptation to stoma surgery : the role of self-efficacy. Psychosom Med 1996; 58: 183-91.

13. Vaizey CJ, Kamm MA, Gold DM, Bartram CI, Halligan S, Nicholls RJ. Clinical, physiological, and radiological study of a new purpose-designed artificial bowel sphincter. Lancet 1998; 351: 105-9.

14. Chandler JG, Adams RB, Friedman CJ, Marcella KL, Guerrant RL. Assessment of implantable ileostomy sphincter. Surgery 1985; 98: 72-80.

15. Heiblum M, Cordoba A. An artificial Sphincter: a preliminary report. Dis Colon Rectum 1978; 21: 562-6.

16. Szinicz G. A new implantable sphincter prothesis for artificial anus. Int J Artif Organs 1980; 3: 358-62.

17. De Paula JB, Cliquet A. A feasibility study on a new artificial sphincter for colostomy. Int J Artif Organs 1995; 19(3): 222-4.

18. Porter JA, Salvati EP, Rubin RJ, Eisenstat TE. Complications of colostomies. Dis Colon Rectum !989; 32: 299-303.

Gomes EGA, Brandt CT, Jucá MJ, Menezes HL. Continent colostomy with artificial sphincter of silicone: study in dogs. Acta Cir Bras [serial online] 2003 Nov-Dec;18(6). Available from URL: http://www.scielo.br/acb.

ABSTRACT - Purpose: This was the rational and the aim of the present investigation. Artificial silicone coated sphincters were implanted in dog's colostomies. It was analyzed the continence for solid and liquid stools as well as the complications from these surgical procedures. Methods: It was used 13 mongrel dogs, eleven males and two females, weights from 16 to $30 \mathrm{~kg}$ ( $22.5 \pm 4.3 \mathrm{~kg}$ ). Terminal colostomies were made using the $\mathrm{C} \mathrm{C}-\mathrm{Silimed}^{\circledR}$ devices, which were activated for an eight-hour periods. The animals were observed for 15 weeks. Results: Fecal continence was proved by barium enema. The pressure in the system varied from 60 to $80 \mathrm{~cm}$ of water $(74.8 \pm 5.0 \mathrm{~cm})$. The liquid volume inserted in the reservoir varied from 5 to $8 \mathrm{ml}(6.9 \pm 0.8 \mathrm{ml})$. Fecal continence was obtained in all animals, however, there was an eventual soiling when abdominal pressure was raised. Wound infection occurs in three dogs $(23.1 \%)$ and intestinal obstruction in one $(7.7 \%)$. Conclusion: The silicone artificial sphincter ( C C-Silimed $\left.{ }^{\circledR}\right)$ was effective for obtaining continence for solid and liquid stools. The results of this investigation give support to the future use of this sphincter in human patients with definitive colostomy.

KEY WORDS - Colostomy. Fecal incontinence. Artificial esphincter.

Conflito de interesse: nenhum

Fonte de financiamento: nenhuma

Correspondência:

Edmundo Guilherme de Almeida Gomes

Rua Barão José Miguel, 126/601

57055-160 Maceio-AL

Tel: (82)338-2481

gomesedmundo@aol.com.

Data do recebimento: 10/09/2003

Data da revisão: 30/09/2003

Data da aprovação: 19/10/2003 